Faculty of Science

Faculty Publications

This is a post-print version of the following article:

Surface Structure of Acrylate Polymer Adhesives

Sandra Roy, Stephan Freiberg, Claude Leblanc, and Dennis K. Hore

January 2017

The final publication is available at ACS Publications via:

https://doi.org/10.1021/acs. langmuir.6b03875

Citation for this paper:

Roy, S.; Freiberg, S.; Leblanc, C.; \& Hore, D. K. (2017). Surface structure of acrylate polymer adhesives. Langmuir, 33(8), 1763-1768. DOI: 10.1021/acs. langmuir.6b03875 


\title{
Surface Structure of Acrylate Polymer Adhesives
}

\author{
Sandra Roy, ${ }^{\dagger}$ Stephan Freiberg, ${ }^{\ddagger}$ Claude Leblanc, ${ }^{\ddagger}$ and Dennis K. Hore ${ }^{*, \dagger}$ \\ $\dagger$ Department of Chemistry, University of Victoria, Victoria, British Columbia, V8W 3V6, \\ Canada \\ $\$$ Mapei Inc., 2900 Avenue Francis-Hughes, Laval, Quebec, H7L 3J5, Canada \\ E-mail: dkhore@uvic.ca
}

\begin{abstract}
Total internal reflection infrared absorption and visible-infrared sum-frequency spectroscopy were used to study the role of acrylic acid in the evolution of surface structure in a poly(butyl acrylate)-based pressure sensitive adhesive during the drying process. By monitoring these spectral responses and calculating the heterospectral correlation coefficients, we have established that acrylic acid alters the nature of the molecular interactions at the surface. In the absence of acrylic acid, butyl acrylate orientation is driven by the packing of the polymer as the water evaporates. When acrylic acid is present a rapid ordering of the copolymer takes place as a result of favorable hydrogen bonding interactions with the surface.
\end{abstract}




\section{Introduction}

Polymeric adhesion is governed by mechanical, physical and chemical properties of the materials employed. ${ }^{1,2}$ In pressure-sensitive adhesives (PSA), adhesion is typically characterized by evaluating their shear, peel, and tack properties; these are directly related to the material performance in the end-use application. Polymers with low glass transition temperatures $\left(T_{g}\right)$ will exhibit higher wettability and thus higher tack force. However, polymeric adhesives with higher $T_{g}$ exhibit higher shear resistance due to reduced chain mobility, thus providing a final material with an increased cohesive strength. Poly(butyl acrylate) is a common PSA base that satisfies the wettability criteria, but does not offer much shear resistance. Copolymerizing with small amounts of acrylic acid is a convenient method to increase the PSA shear properties through hydrogen bonding while maintaining the low $T_{g}$ characteristics of the base poly(butyl acrylate). ${ }^{3,4}$ The preparation of these adhesives involves drying them on a surface (the backing material) prior to use. The characteristic mechanical properties develop during the drying process. Studying PSA drying therefore can offer insight into how the components of the film interact to yield the desired properties. ${ }^{5,6}$ Although the macroscopic and mechanical properties are routinely measured, the behavior and contribution of acrylic acid upon drying and hydrogen bond formation is not understood on a microscopic scale. ${ }^{7}$

Vibrational spectroscopy may be used as an in situ probe of PSA structure. In an attenuated total internal reflection (ATR) IR absorption experiment, the probe depth is limited to the evanescent wave penetration. Depending on the substrate, wavelength, and angle of incidence, this is typically around $1 \mu \mathrm{m}$. ATR-IR is therefore ideally suited to probe the concentration of various components near the surface. Visible-infrared sumfrequency generation $(\mathrm{SFG})$ is a nonlinear vibrational technique that is more surface-specific

on account of the requirement of broken centrosymmetry. ${ }^{8-10}$ In systems such as polymers, this symmetry condition is typically found only immediately adjacent to the interface. SFG is therefore established as a sensitive probe of polymer surface structure. ${ }^{11-24}$ However, it is 
often challenging to separate the effects of orientation from changes in concentration in the SFG signal. In this study we will therefore combine IR absorption and SFG spectroscopy to study the drying process of butyl acrylate PSAs containing varying amounts of acrylic acid. Quantitative comparison of the IR and SFG spectra in the form of heterospectral 2D correlation maps offers addition insight into the role of acrylic acid in the polymer-surface interaction.

\section{Methods}

Materials. Monomer preparations of $0 \%, 2 \%$ and $4 \% \mathrm{wt} / \mathrm{wt}$ of acrylic acid with butyl acrylate were vigorously agitated with the required amount of water, non-ionic surfactant (aliphatic alcohol ethoxylate) and ionic surfactant (sulfated nonyl phenol ethoxylate) to make the pre-emulsion. Five percent of the pre-emulsion was charged to the reactor with a known amount of water and non-ionic surfactant. The reaction was initiated by addition of sodium persulfate to the reactor at $72^{\circ} \mathrm{C}$ under constant stirring at $120 \mathrm{rpm}$. A temperature exotherm indicated when polymerization initiation was underway and the main pre-emulsion feed was started 10 min after the initial sodium persulate drop. A second sodium persulfate solution was simultaneously fed into the reactor with the main pre-emulsion feed over $3 \mathrm{~h}$ and the reaction temperature was maintained between $75-80^{\circ} \mathrm{C}$. The sodium persulfate feed was allowed to continue for another 30 min after termination of the main pre-emulsion feed so as to eliminate the unreacted monomers. The resulting latexes were cooled to room temperature by the jacketed reactor and filtered using a 60-mesh screen. Each latex was neutralized to about pH 7 using a $14 \%$ ammonia solution. The result was between $49.5-50.5 \%$ solids with $\mathrm{pH}$ values ranging from 6.9-7.1, and viscosities less than $500 \mathrm{cP}$. The residual monomer content was measured to be less than 300 ppm, the particle sizes were in the range of $300 \mathrm{~nm}$, and the filtered coagulate content was less than $0.25 \%$. In all experiments, solutions were deposited onto the substrates to thickness of $2 \mathrm{~mm}$ prior to drying. IR and SFG drying 
experiments were conducted at $21^{\circ} \mathrm{C}$ and $40 \%$ relative humidity.

IR absorption spectroscopy. Polarized ATR-IR spectra were collected on a Bruker Vertex 70 with a $\mathrm{KBr}$ beamsplitter, wire-grid polarizer on a $\mathrm{BaF}_{2}$ substrate (Thorlabs, Newton NJ) and a deuterated triglycine sulfate (DTGS) detector. s- and p-polarized spectra were acquired as a result of 200 co-additions at $2 \mathrm{~cm}^{-1}$ resolution, and $6 \mathrm{~mm}$ aperture size. A $4 \mathrm{~mm}$ Ge internal reflecting element (Pike Technologies, Madison WI) cut at $45^{\circ}$ resulted in 19 internal reflections, 9 of which were TIR at the lower Ge-air interface, and 10 of which were ATR at the top Ge-PSA interface. Drying was monitored over $10 \mathrm{~h}$, collecting spectra ever 30 min. Post-processing included Norton-Beer apodization, 4 levels of zerofilling, and a standard ATR correction to provide the transmission-equivalent absorption coefficient proportional to the imaginary component of the linear susceptibility $\chi^{(1)} \cdot{ }^{25}$ No evidence of anisotropy was found over the evanescent wave penetration depth of the mid-IR beam, so only p-polarized spectra are considered for subsequent analysis as they displayed higher absorbance.

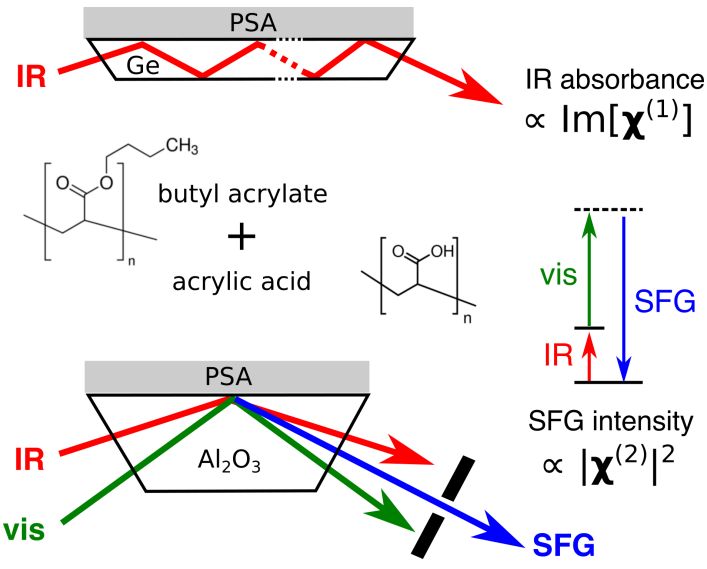

Figure 1: In ATR-IR absorption spectroscopy, the reflectance of an IR probe beam is altered by transfer of energy from the evanescent wave into the PSA in contact with the Ge prism. In visible-infrared SFG spectroscopy, a new colour of light is created from regions of the PSA-sapphire interface where molecules are oriented in a polar manner.

Visible-infrared sum-frequency spectroscopy. A more structurally-sensitive probe of the substrate-PSA interface was provided by a nonlinear vibrational method, visibleinfrared sum-frequency spectroscopy. Here a p-polarized visible laser $\left(\omega_{\text {vis }}=18,797 \mathrm{~cm}^{-1}\right)$ 
produced from a frequency-doubled Nd:YAG laser (Ekspla PL2241) 22 ps pulse width, $10 \mathrm{~Hz}$ repetition rate, $100 \mu \mathrm{J} /$ pulse, $\theta_{\text {vis }}=70^{\circ}$ incident angle was spatially and temporally overlapped at the PSA-sapphire interface with a p-polarized mid-IR beam generated in a parametric process (Ekspla PG501), tunable from $\omega_{\mathrm{IR}}=2800-3750 \mathrm{~cm}^{-1}$, nominally $200 \mu \mathrm{J} /$ pulse, $\theta_{\mathrm{IR}}=65^{\circ}$ incidence. Sapphire (Del Mar Photonics, San Diego CA) was chosen as the internal reflecting element as its high index of refraction enabled us to stay above the critical angle during the polymer drying process. The p-polarized component of the reflected SFG beam was focused onto the $0.5 \mathrm{~mm}$ entrance slit of a $500 \mathrm{~mm}$ spectrometer (Acton SP2500i) fitted with a 600 groove/mm grating blazed at $500 \mathrm{~nm}$. Spectra were collected using a CCD camera (Princeton Instruments Pixis 400B) operating at $-80^{\circ} \mathrm{C}$, averaging 200 laser pulses at each IR wavelength (2 s exposure, 10 averages). As a result of local vibrational mode symmetry, some modes are more prominent in certain polarization schemes. This all p-polarized (ppp) excitation and collection scheme was employed as it generated the most SFG signal and displayed the largest number of vibrational modes.

In general, the polarization of a material $P$ may be expressed in terms of susceptibility tensor elements

$$
P_{i}=\varepsilon_{0}\left(\chi_{i j}^{(1)} E_{j}+\frac{1}{2} \chi_{i j k}^{(2)} E_{j} E_{k}+\cdots+\frac{1}{n !} \chi^{(n)} E^{n}\right)
$$

where $\chi^{(n)}$ is the $n$-th order susceptibility, $E$ is the electric component of the electromagnetic field, and $\varepsilon_{0}$ is the vacuum permittivity. Here $i, j, k$ refer to any of the laboratory-frame Cartesian $x, y$ or $z$ components. The linear susceptibility $\chi^{(1)}$ is responsible for IR absorption in the ATR-IR experiment. If there are any regions of the material that lack inversion symmetry (an imbalance in chemical functional groups pointing towards the surface and towards the bulk PSA phase), then $\chi^{(2)} \neq 0$ and a three-wave mixing process occurs in which one visible and one infrared photon are annihilated to give rise to a new photon. Conservation of energy tells us to search for this light at the sum-frequency, $\omega_{\mathrm{SF}}=\omega_{\mathrm{vis}}+\omega_{\mathrm{IR}}$, and in the momentum-conserving direction determined from $\theta_{\mathrm{vis}}$ and $\theta_{\mathrm{IR}}$. If light is detected at $\omega_{\mathrm{SF}}$, we know that the PSA components must be oriented in such a way that their up/down 
symmetry has been broken. In the SFG spectra, the amount of p-polarized light generated at this new frequency is plotted as a function of $\omega_{\mathrm{IR}}$, hence yielding a surface vibrational spectrum. These spectra were collected every hour during the drying process.

Heterospectral correlation analysis. Two-dimensional correlation analysis of spectral data was first introduced by Noda as a powerful means of qualitatively and quantitatively examining changes that occur between two spectral variables upon the action of an external perturbation, most commonly time. ${ }^{26-30} \mathrm{~A}$ subsequent development in this field was the introduction of heterospectral correlation, allowing the effect of the same perturbation to be studied on two different data sets, offering the possibility of using orthogonal or otherwise complementary characterization techniques. ${ }^{31-36}$

We have recently demonstrated that heterospectral correlation analysis may be applied to ATR-IR and SFG data of binary mixtures of ethanol and water as a function of the bulk ethanol mole fraction, providing insight into the mechanism of alcohol adsorption onto silica surfaces. ${ }^{25}$ Although it is advantageous to have $\operatorname{Im}\left[\chi^{(2)}\right]$ data available for such analysis, we have also demonstrated that it is possible to use $\left|\chi^{(2)}\right|^{2}$ spectra directly. ${ }^{37}$ This is convenient for systems such as the buried PSA-substrate interface for which interferometric SFG techniques are not readily applied. Here we use the magnitude $\left|\chi^{(2)}\right|$ as it is proportional to the concentration of molecules that contribute to the SFG signal. In the 2D heterospectral analysis, the complex-valued cross-correlation coefficient $\Phi\left(\omega, \omega_{\mathrm{IR}}\right)+i \Psi\left(\omega, \omega_{\mathrm{IR}}\right)$ is calculated where $\omega$ is the wavenumber in the IR absorption experiment, and $\omega_{\mathrm{IR}}$ is the IR wavenumber in the IR+vis SFG experiment. The first step in the analysis is to prepare the mean-shifted spectra

$$
\begin{aligned}
\operatorname{Im}\left[\chi_{i}^{(1)}(\omega)\right] & =\operatorname{Im}\left[\chi^{(1)}\left(\omega, t_{i}\right)\right]-\frac{1}{m} \sum_{i=1}^{m} \operatorname{Im}\left[\chi^{(1)}\left(\omega, t_{i}\right)\right] \\
\left|\chi_{i}^{(2)}\left(\omega_{\mathrm{IR}}\right)\right| & =\left|\chi^{(2)}\left(\omega_{\mathrm{IR}}, t_{i}\right)\right|-\frac{1}{m} \sum_{i=1}^{m}\left|\chi^{(2)}\left(\omega_{\mathrm{IR}}, t_{i}\right)\right|
\end{aligned}
$$

where $\operatorname{Im}\left[\chi^{(1)}\right]$ comes from the IR absorption data, $\left|\chi^{(2)}\right|$ is obtained from the square root of 
the SFG intensity data, and $m$ refers to the number of time points at which each spectrum was acquired. For a $10 \mathrm{~h}$ drying experiment with spectra recorded each hour, $m=10$. Each set of mean-shifted spectra was then arranged into a matrix with the intensity at each wavenumber in the columns, and the spectra acquired at different time points in the rows. For example, the matrix of mean-shifted IR absorption spectra was constructed as

$$
\operatorname{Im}\left[\chi^{(1)}(\omega)\right]=\left[\begin{array}{c}
\operatorname{Im}\left[\chi_{1}^{(1)}(\omega)\right] \\
\operatorname{Im}\left[\chi_{2}^{(1)}(\omega)\right] \\
\operatorname{Im}\left[\chi_{3}^{(1)}(\omega)\right] \\
\vdots \\
\operatorname{Im}\left[\chi_{m}^{(1)}(\omega)\right]
\end{array}\right]
$$

and an analogous matrix was constructed from the SFG data, which we refer to as $\left|\chi^{(2)}\left(\omega_{\mathrm{IR}}\right)\right|$. The real or synchronous component $\Phi$ is then determined from

$$
\Phi\left(\omega, \omega_{\mathrm{IR}}\right)=\frac{1}{m-1} \operatorname{Im}\left[\chi^{(1)}(\omega)\right]^{T} \cdot\left|\chi^{(2)}\left(\omega_{\mathrm{IR}}\right)\right|
$$

where the superscript $T$ refers to the matrix transpose. The imaginary or asynchronous component of the correlation coefficient $\Psi$ is obtained from

$$
\Psi\left(\omega, \omega_{\mathrm{IR}}\right)=\frac{1}{m-1} \operatorname{Im}\left[\chi^{(1)}(\omega)\right]^{T} \cdot N \cdot\left|\chi^{(2)}\left(\omega_{\mathrm{IR}}\right)\right|
$$

where $N$ is the Hilbert-Noda transformation matrix

$$
\boldsymbol{N}=\frac{1}{\pi}\left[\begin{array}{ccccc}
0 & 1 & \frac{1}{2} & \frac{1}{3} & \ldots \\
-1 & 0 & 1 & \frac{1}{2} & \ldots \\
-\frac{1}{2} & -1 & 0 & 1 & \ldots \\
-\frac{1}{3} & -\frac{1}{2} & -1 & 0 & \ldots \\
\ldots & \ldots & \ldots & \ldots & \ldots
\end{array}\right]
$$


which may be calculated according to

$$
N_{j k}= \begin{cases}0, & \text { if } j=k \\ 1 /[\pi(k-j)], & \text { otherwise. }\end{cases}
$$

\section{Results and Discussion}

IR absorption spectroscopy. Figure 2 displays the infrared spectra collected during the PSA drying process. The spectra show an overall decrease in the $\mathrm{OH}$ stretching region (3100-3600 $\left.\mathrm{cm}^{-1}\right)$ and an overall increase in the $\mathrm{CH}$ region region $\left(2800-3000 \mathrm{~cm}^{-1}\right)$. For the $0 \%$ acrylic acid content PSA, the decrease in $\mathrm{OH}$ stretching signal can be assigned to drying, i.e. the removal of the water. The increase in $\mathrm{CH}$ intensity is most likely due to the increase in polymer density when water molecules are removed. Such evidence of increased packing is present in all samples. However, for the $2 \%$ and $4 \%$ acrylic acid content PSA, the $\mathrm{OH}$ stretching band now has two contributors: water and acrylic acid. While the water $\mathrm{OH}$ contribution decreases during drying, the acrylic acid $\mathrm{OH}$ contribution increases due to enhanced packing. This explains why the signal in this range is still present after $10 \mathrm{~h}$.

Visible-infrared sum-frequency spectroscopy. Inspecting the SFG spectra in Figure 3, certain trends can be observed. In all samples, the $\mathrm{OH}$ stretching region near $3700 \mathrm{~cm}^{-1}$ shows an increase in intensity during the drying process. This peak has previously been assigned to the sapphire surface free $\mathrm{OH}$ group, ${ }^{38-40}$ where it appeared around $3700 \mathrm{~cm}^{-1}$ when not hydrogen bonded, and closer to $3500 \mathrm{~cm}^{-1}$ when interacting with its environment (through hydrogen bonding or dipole-dipole interactions). During the drying, such free $\mathrm{OH}$ is created as water leaves. This band has also been observed in SFG spectra of other polymers at the sapphire surface. ${ }^{38,39}$ For the $0 \%$ acrylic acid sample, the H-bonded OH region (3050$3500 \mathrm{~cm}^{-1}$ ) shows a constant decreases after the first hour, indicating that water is leaving. We attribute the overall increase in intensity during the first hour to the substantial increase in the dominant $3700 \mathrm{~cm}^{-1}$ sapphire $\mathrm{OH}$ band. For the other two samples, the acrylic acid 

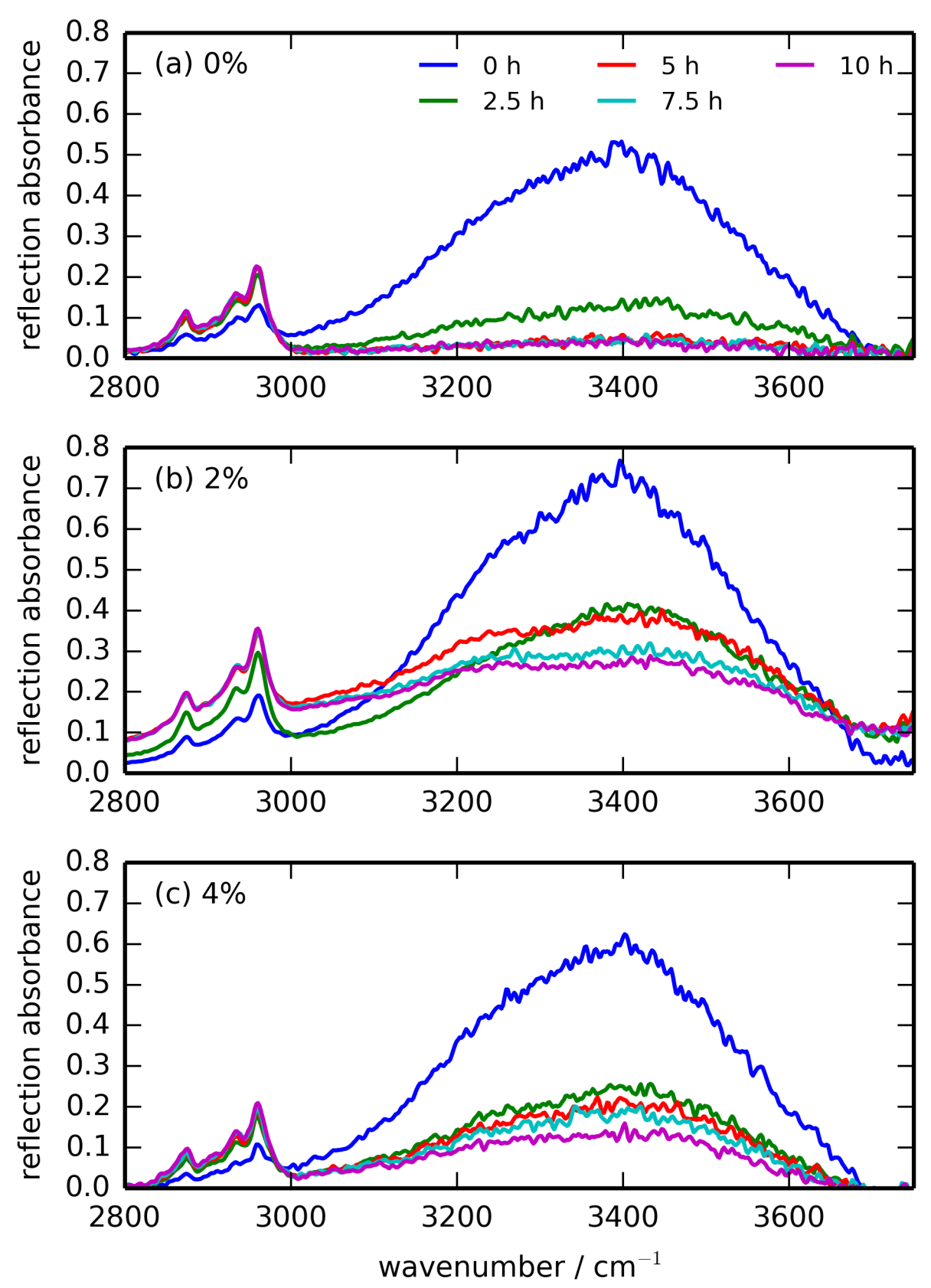

Figure 2: A subset of the reflection absorbance spectra (collected every $30 \mathrm{~min}$ for $10 \mathrm{~h}$ ) as measured in the ATR-IR experiment following a simple ATR correction that renders the data proportional to $\operatorname{Im}\left[\chi^{(1)}\right]$ for the sample containing (a) no acrylic acid, (b) $2 \%$ acrylic acid and (c) $4 \%$ acrylic acid as a function of the drying time as indicated in the inset to the top panel. 

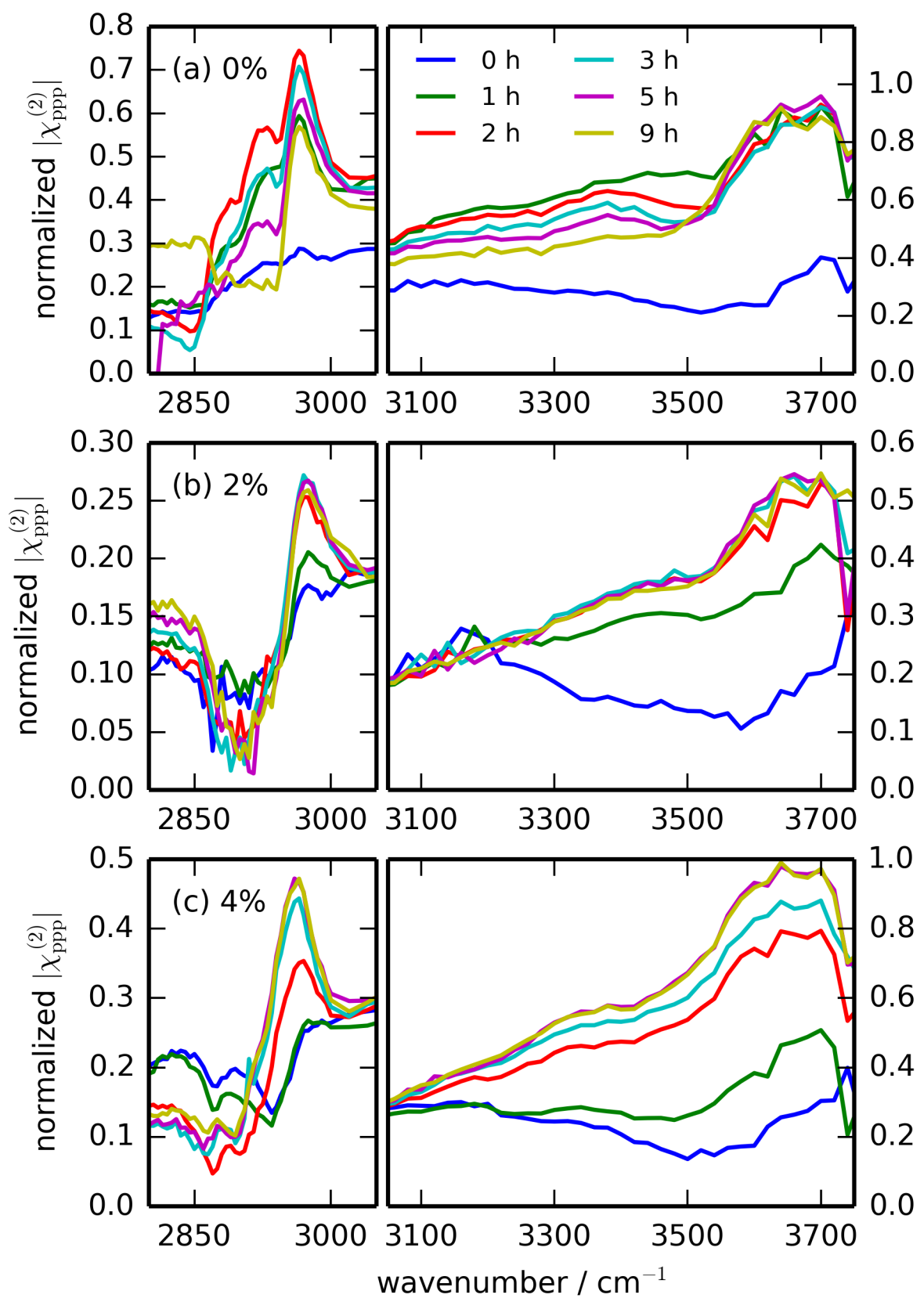

Figure 3: A subset of the $\left|\chi_{\mathrm{ppp}}^{(2)}\right|$ spectra (collected every hour for $10 \mathrm{~h}$ ) as measured in the SFG experiment for the sample containing (a) no acrylic acid, (b) $2 \%$ acrylic acid and (c) $4 \%$ acrylic acid as a function of the drying time as indicated in the inset to the top panel. The axes are broken at $3050 \mathrm{~cm}^{-1}$ so as to magnify the $\mathrm{CH}$ stretching region in the 2800-3050 $\mathrm{cm}^{-1}$ region. 
present also contributes to the spectral intensity in that region. In the case of the $2 \%$ sample, this results in very little change as the water leaves. The $4 \%$ sample exhibits an increase in the hydrogen bonded intensity. Overall, this points to a trend where, once dry, the acrylic acid content is revealed by the ratio of hydrogen-bonded $\left(3050-3500 \mathrm{~cm}^{-1}\right)$ to free $\mathrm{OH}$ signal $\left(3700 \mathrm{~cm}^{-1}\right)$ in the spectra. The $\mathrm{CH}$ stretching region $\left(2800-3050 \mathrm{~cm}^{-1}\right)$ shows many features that evolve differently during the drying process, but it is difficult to interpret these results in the raw SFG spectra as a result of the congestion, and the co-dependence of density and orientation in the $\chi^{(2)}$ process. IR-SFG heterospectral correlation is ideally suited to analyze this spectral region.

Heterospectral correlation analysis. Figure 4 shows the synchronous (top row) and asynchronous (bottom row) IR-SFG heterospectral correlation of the three materials in the drying process. The intensity of peaks in the synchronous map are related to the strength of the correlation, while the sign indicates whether the IR and SFG spectral features are both increasing or decreasing (positive, red contours) or trending in opposite directions (negative, blue contours). For the $0 \%$ acrylic acid content, the synchronous map (Figure 4a) obtained for spectra collected after the initial hour reveals a strong correlation between all $\mathrm{CH}$ modes in the IR and SFG spectra. As these features are negative, this indicates that the IR $\mathrm{CH}$ modes are increasing in intensity while the SFG signals are decreasing during the drying process. This is strongly indicative of an orientation change, as the IR signal is rather insensitive to the molecular orientation. The corresponding asynchronous map may be used to assess the sequence of events, i.e. if the changes as a function of time occur concomitantly (zero or minimal asynchronous signal) or with time lag (appreciable signal). The inset on the asynchronous map indicates the percentage of the largest asynchronous signal relative to the largest synchronous signal. This is an important feature to note, as it indicates whether there is an appreciable asynchronous component to the correlation. In the asynchronous map of the $0 \%$ sample (Figure $4 \mathrm{~b}$ ), we can notice a strong negative feature throughout, up to $36 \%$ of the synchronous signal. To interpret the asynchronous map, one must take in 
consideration the sign of both synchronous and asynchronous peaks. According to Noda's rules, ${ }^{26}$ if both show the same sign for a specific cross peak, then the change in IR intensity occurs prior to the change in SFG intensity. (If the signs are opposite, then the conclusions are reversed.) For this no-acrylic acid sample, the negative asynchronous feature together with the negative corresponding feature in synchronous map indicates that there is a delay where the decrease in the SFG occurs after the increase in IR CH stretching mode intensities. The increase in interfacial PSA density, seen in the IR, precedes the ordering of the interfacial PSA seen in the SFG. Taking into consideration the strong increase of alkyl stretching SFG intensity in the first hour, we can speculate on the order of events. During the first hour, the SFG intensity increase is mostly due to a larger number of molecules at the interface, then the density increase continues, causing a more tightly packed layer consequently producing a stronger ordering of the polymer. In summary, the ordering of the PSA with 0\% acrylic acid content is likely driven by the packing of the poly(butyl acrylate).

For the $2 \%$ acrylic acid content PSA, the largest SFG peak at $2970 \mathrm{~cm}^{-1}$ and all of the CH IR modes have positive cross peaks in the synchronous map (Figure 4c). This reflects the increase in signal of both SFG and IR modes as the sample dries. The asynchronous map (Figure 4d) shows a strong negative feature in this same region. Coupled with the positive synchronous signal and the high asynchronous intensity (up to $54 \%$ of the corresponding synchronous map), this signifies that the major change in SFG precedes most of the changes in the IR signal in this region. From this we conclude that there is an orientation change prior to the increase in density. It is conceivable that the polymer would reorient itself rapidly for the acrylic acid to hydrogen bond with the sapphire surface $\mathrm{OH}$ as the water content decreases. This phenomena may also be observed in the hydrogen bonded $\mathrm{OH}$ region of the SFG spectra (3300-3500 $\left.\mathrm{cm}^{-1}\right)$, as most of the increase in intensity occurs during the first $2 \mathrm{~h}$.

For the $4 \%$ acrylic acid content sample, the synchronous map (Figure 4e) shows a strong positive feature between all IR $\mathrm{CH}$ modes and the predominant SFG $\mathrm{CH}$ mode. This is 

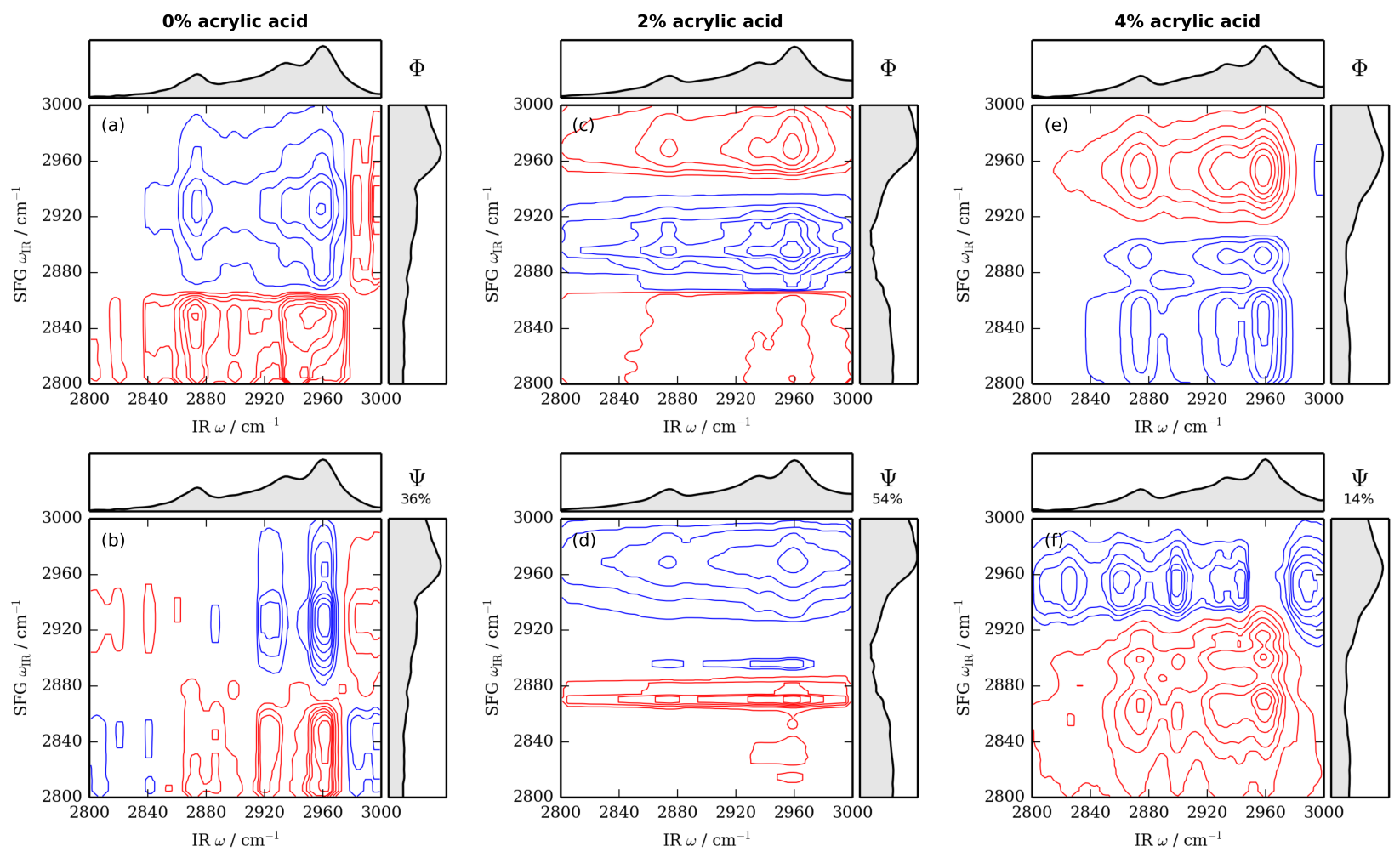

Figure 4: Heterospectral synchronous ( $\Phi$, top row a,c,e) and asynchronous ( $\Psi$, bottom row b,d,f) correlation maps that relate SFG to IR spectral changes during the drying process. Results are shown for (a,b) 0\% acrylic acid, (c,d), 2\% acrylic acid, and (e,f) 4\% acrylic acid. Positive contours are drawn in red; negative contours in blue. Percentages in the inset of the $\Psi$ plots indicate the fraction of the largest synchronous signal found in the corresponding asynchronous plot.

due to a increase in both spectral intensities while drying. From the corresponding negative feature in the asynchronous map (Figure 4f), we can determine that, as in the case of the $2 \%$ acrylic acid sample, the change in the SFG precedes the change in the IR. However, if we take into consideration that the largest asynchronous signal is less than $15 \%$ of the synchronous response, it is clear that, for the $4 \%$ sample, changes in the IR and SFG are more closely linked to each other in time then for the other samples. This indicates that any significant orientation changes in the polymer occur faster than our measurement timescale. During the $10 \mathrm{~h}$ drying experiment, the increase in SFG signal is mostly due to an increase of number of molecules at the interface. This can also be explained by the strong hydrogen bonding possibilities with the sapphire surface. Further clues come from the steady increase 
in the SFG water hydrogen bonding region. The similar concomitant increase in $\mathrm{CH}$ and $3300-3500 \mathrm{~cm}^{-1} \mathrm{OH}$ modes is evidence of an increase in density of acrylic acid with no significant reordering for this system.

\section{Conclusions}

We have used a combination of IR absorption and nonlinear vibrational spectroscopy to study the interaction of acrylic acid-containing butyl acrylate pressure-sensitive adhesive polymers with a sapphire surface. All of the data point to a picture of the PSA drying process that is dependent on the acrylic acid content. In the absence of acrylic acid, the poly(butyl acrylate) orientation is dictated by the packing, related to the water content. This packing requirement lengthens the time required to achieve optimal orientation at the surface as significant drying is required. When a small amount of acrylic acid is added, the orientation of the polymer is dictated by hydrogen-bonding interactions. As a result, reorientation occurs on a timescale that is much faster than the change in density. Such insight obtained during the drying process can lead to a better understanding, and a molecular rationalization, of the final PSA mechanical properties.

Notes. The authors declare no competing financial interest.

\section{Acknowledgement}

We thank the Natural Sciences and Engineering Research Council of Canada (NSERC) for support of this science with Discovery, Interaction, and Engage grants. We thank Fabien Perineau (Mapei Inc.) for helpful discussion. 


\section{References}

(1) Sharif, A.; Mohammadi, N.; Nekoomanesh, M.; Jahani, Y. The Role of Interfacial Interactions and Loss Function of Model Adhesives on Their Adhesion to Glass. J. Adhes. Sci. Technol. 2002, 16, 33-45.

(2) Awaja, F.; Gilbert, M.; Kelly, G.; Fox, B.; Pigram, P. J. Adhesion of Polymers. Prog. Polym. Sci. 2009, 34, 948-968.

(3) Ghim, D.; Kim, J. H. Effects of Composition and Layer Thickness of a Butyl Acrylate/Acrylic Acid Copolymer on the Adhesion Properties. Korean J. Chem. Eng. 2016, 33, 707-710.

(4) Tobing, S. D.; Klein, A. Molecular Parameters and Their Relation to the Adhesive Performance of Acrylic Pressure-Sensitive Adhesives. J. Appl. Polym. Sci. 2000, 79, $2230-2244$.

(5) Steward, P. A.; Hearn, J.; Wilkinson, M. C. An overview of polymer latex film formation and properties. Adv. Colloid Interface Sci. 2000, 86, 195-267.

(6) Emamieh, S.; Sadr, A.; Ghasemi, A.; Torabzadeh, H.; Akhavanzanjani, V.; Tagami, J. Effects of Solvent Drying Time and Water Storage on Ultimate Tensile Strength of Adhesives. Dental Biomater. 2014, 5, 51-57.

(7) Kiil, S. Drying of Latex Films and Coatings: Reconsidering the Fundamental Mechanisms. Prog. Org. Coatings 2006, 57, 236-250.

(8) Shen, Y. R. Basic Theory of Surface Sum-Frequency Generation. J. Phys. Chem. C 2012, 116, 15505-15509.

(9) Lambert, A. G.; Davies, P. B.; Neivandt, D. J. Implementing the Theory of Sum Frequency Generation Vibrational Spectroscopy: A Tutorial Review. Appl. Spectrosc. Rev. 2005, 40, 103-145. 
(10) Shen, Y. R. Surface Properties Probed by Second-Harmonic and Sum-Frequency Generation. Nature 1989, 337, 519-525.

(11) Chen, Z.; Shen, Y. R.; Somorjai, G. A. Studies of Polymer Surfaces by Sum Frequency Generation Vibrational Spectroscopy. Annu. Rev. Phys. Chem. 2002, 53, 437-465.

(12) Wang, M.; Li, B.; Chen, Z.; Lu, X. Molecular-Level Structures at Poly(4-vinyl pyridine)/Acid Interfaces Probed by Nonlinear Vibrational Spectroscopy. J. Polym. Sci. B Polym. Phys. 2016, 54, 848-852.

(13) Chen, Z. Investigating Buried Polymer Interfaces Using Sum Frequency Generation Vibrational Spectroscopy. Prog. Polym. Sci. 2010, 35, 1376-1402.

(14) Myers, J. N.; Chen, Z. Polymer Molecular Behaviors at Buried Polymer/Metal and Polymer/Polymer Interfaces and Their Relations to Adhesion in Packaging. J. Adhes. 2016, $0,1-23$.

(15) Loch, C. L.; Ahn, D.; Chen, Z. Sum Frequency Generation Vibrational Spectroscopic Studies on a Silane Adhesion-Promoting Mixture at a Polymer Interface. J. Phys. Chem. B 2006, 110, 914-918.

(16) Loch, C. L.; Ahn, D.; Chen, C.; Wang, J.; Chen, Z. Sum Frequency Generation Studies at Poly(Ethylene Terephthalate)/Silane Interfaces: Hydrogen Bond Formation and Molecular Conformation Determination. Langmuir 2004, 20, 5467-5473.

(17) Chen, C.; Wang, J.; Even, M. A.; Chen, Z. Sum Frequency Generation Vibrational Spectroscopy Studies on Buried Polymer/Polymer Interfaces. Macromolecules 2002, 35, 8093-8097.

(18) Kitano, H. Characterization of Polymer Materials Based on Structure Analyses of Vicinal Water. Polym. J. 2016, 48, 15-24. 
(19) Balzerowski, P.; Meister, K.; Versluis, J.; Bakker, H. J. Heterodyne-Detected Sum Frequency Generation Spectroscopy of Polyacrylic Acid at the Air/Water Interface. Phys. Chem. Chem. Phys. 2016, 18, 2481-2487.

(20) Gautam, K. S.; Schwab, A. D.; Dhinojwala, A.; Zhang, D.; Dougal, S. M.; Yeganeh, M. S. Molecular Structure of Polystyrene at Air/Polymer and Solid/Polymer Interfaces. Phys. Rev. Lett. 2000, 85, 3854-3857.

(21) Harp, G. P.; Gautam, K. S.; Dhinojwala, A. Probing Polymer/Polymer Interfaces. J. Am. Chem. Soc. 2002, 124, 7908-7909.

(22) Rangwalla, H.; Dhinojwala, A. Probing Hidden Polymeric Interfaces Using IR-Visible Sum-Frequency Generation Spectroscopy. J. Adhesion 2004, 80, 37-59.

(23) Rao, A.; Rangwalla, H.; Varshney, V.; Dhinojwala, A. Structure of Poly(Methyl Methacrylate) Chains Adsorbed on Sapphire Probed Using Infrared-Visible Sum Frequency Generation Spectroscopy. Langmuir 2004, 20, 7183-7188.

(24) Choi, H. C.; Ryu, S. R.; Ji, H.; Kim, S. B.; Noda, I.; Jung, Y. M. TwoDimensional Heterospectral Correlation Analysis of X-Ray Photoelectron Spectra and Infrared Spectra For Spin-Coated Films of Biodegradable Poly(3-hydroxybutyrate-co3-hydroxyhexanoate) Copolymers. J. Phys. Chem. B 2010, 114, 10979-10985.

(25) Roy, S.; Covert, P. A.; Jarisz, T. A.; Chan, C.; Hore, D. K. Surface-Bulk Vibrational Correlation Spectroscopy. Anal. Chem. 2016, 88, 4682-4691.

(26) Noda, I.; Ozaki, Y. Two-Dimensional Correlation Spectroscopy: Applications in Vibrational and Optical Spectroscopy; John Wiley \& Sons, Ltd: San Francisco, 2004.

(27) Noda, I. Two-Dimensional Infrared Spectroscopy. J. Am. Chem. Soc. 1989, 111, 81168118. 
(28) Noda, I. Two-Dimensional Infrared (2D IR) Spectroscopy: Theory and Applications. Appl. Spectrosc. 1990, 44, 550-561.

(29) Noda, I. Two-Dimensional Infrared (2D IR) Spectroscopy of Synthetic and Biopolymers. Bull. Am. Phys. Soc. 1986, 31, 520-524.

(30) Noda, I. Close-Up View on the Inner Workings of Two-Dimensional Correlation Spectroscopy. Vibr. Spectrosc. 2012, 60, 146-153.

(31) Noda, I.; Liu, Y.; Ozaki, Y. Two-Dimensional Correlation Spectroscopy Study of Temperature-Dependent Spectral Variations of $N$-Methylacetamide in the Pure Liquid State. 2. Two-Dimensional Raman and Infrared-Raman Heterospectral Analysis. J. Phys. Chem. 1996, 100, 8674-8680.

(32) Kim, H. J.; Kim, S. B.; Kim, J. K.; Jung, Y. M. Two-Dimensional Heterospectral Correlation Analysis of Wide-Angle X-ray Scattering and Infrared Spectroscopy for Specific Chemical Interactions in Weakly Interacting Block Copolymers. J. Phys. Chem. 2006, 110, 23123-23129.

(33) Jung, Y. M.; Czarnik-Matusewicz, B.; Ozaki, Y. Two-Dimensional Infrared, TwoDimensional Raman, and Two-Dimensional Infrared and Raman Heterospectral Correlation Studies of Secondary Structure of $\beta$-Lactoglobulin in Buffer Solutions. $J$. Phys. Chem. B 2000, 104, 7812-7817.

(34) Park, Y.; Noda, I.; Jung, Y. M. Two-Dimensional Correlation Spectroscopy in Polymer Study. Front. Chem. 2015, 3, 14.

(35) Genkawa, T.; Watari, M.; Nishii, T.; Suzuki, M.; Ozaki, Y. Two-Dimensional Heterospectral Correlation Analysis of Water and Liquid Oleic Acid Using an Online Near-Infrared/Mid- Infrared Dual-Region Spectrometer. Appl. Spectrosc. 2013, 67, 724-730. 
(36) Zou, C.; Larisika, M.; Nagy, G.; Srajer, J.; Oostenbrink, C.; Chen, X.; Knoll, W.; Liedberg, B.; Nowak, C. Two-Dimensional Heterospectral Correlation Analysis of the Redox-Induced Conformational Transition in Cytochrome c Using Surface-Enhanced Raman and Infrared Absorption Spectroscopies on a Two-Layer Gold Surface. J. Phys. Chem. B 2013, 117, 9606-9614.

(37) Roy, S.; Post, J. S.; Hung, K.-K.; Stege, U.; Hore, D. K. 2D Correlation Analysis in Vibrational Sum-Frequency Generation Spectroscopy. J. Mol. Struct. 2014, 1069, 103-111.

(38) Zhou, J.; Anim-Danso, E.; Zhang, Y.; Zhou, Y.; Dhinojwala, A. Interfacial Water at Polyurethane-Sapphire Interface. Langmuir 2015, 31, 12401-12407.

(39) Nanjundiah, K.; Hsu, P. Y.; Dhinojwala, A. Understanding Rubber Friction in the Presence of Water Using Sum-frequency Generation Spectroscopy. J. Chem. Phys. 2009, 130, 024702 .

(40) Zhu, H.; Dhinojwala, A. Thermal Behavior of Long-Chain Alcohols on Sapphire Substrate. Langmuir 2015, 31, 6306-6313. 


\section{Graphical TOC Entry}

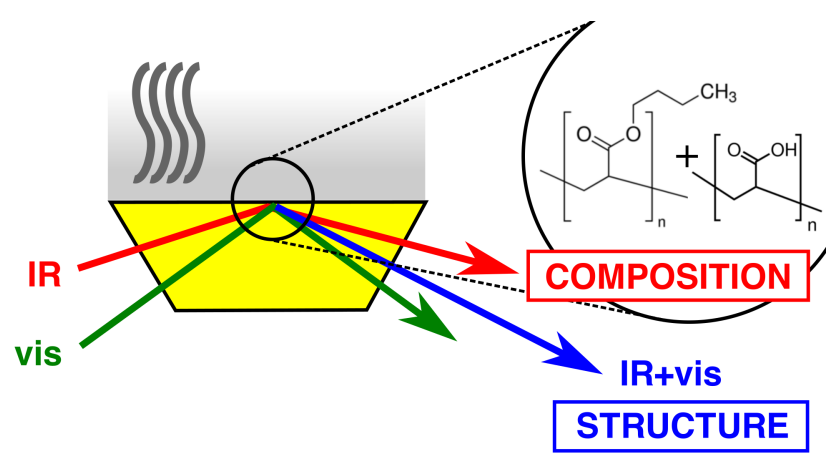

Table of contents graphic, 3.25 in $\times 1.75$ in. 